http://dx.doi.org/10.12795/RAA.2016.11.03

\title{
LA REFORMA LABORAL DE 2012 Y EL AUMENTO DEL DESPIDO Y DESEMPLEO EN ESPAÑA
}

\author{
Ubaldo Martínez Veiga
}

Universidad Nacional de Educación a Distancia (UNED)

\section{Resumen.}

Se va a partir del conjunto de leyes que se han promulgado por el gobierno del Partido Popular desde el 10 de febrero de 2012 hasta el 27 de febrero de 2013, y se va a tratar de demostrar, con el análisis de los textos legales y de las consecuencias prácticas que han acarreado cómo se trata de la imposición por parte de una clase dominante de unas determinaciones legales de carácter neoliberal que perpetúan la división de clase.

La tesis de este trabajo consiste en descubrir cómo la legislación que constituye lo que algunos denominan la "Reforma Laboral del Partido Popular" ha servido en primer lugar para dar mayor poder al empresariado y a la vez ha traído como consecuencia la simplificación y facilitación del despido, con lo cual ha favorecido, de hecho, el despido en tiempos de crisis que es un recurso utilizado muy frecuentemente por los empresarios.

Desde este punto de vista, se prueba, que sin entrar en la relación entre legislación laboral y empleo, lo que sí se prueba es la relación entre esta legislación y el despido y desempleo.

\section{Palabras Claves.}

Flexibilidad externa, Flexibilidad interna, despido, empleo, desempleo, legislación laboral. 


\begin{abstract}
.
Starting from the laws passed by the Popular Party between 10 feb. 2012 and 27 feb. 2013 , this is an attempt to demonstrate through legal text analysis and their practical consequences, how the dominant class has imposed legal determinants that are neoliberal in character to perpetuate class divisions.

The thesis here discovers how this Labour Reform Legislation empowers business more and thus simplifies and facilitates job loss. In times of crisis this is a resource frequently used by them which proves the relation between this legislation and firing and unemployment.
\end{abstract}

\title{
Keywords.
}

External flexibility, internal flexibility, dismissal, employment, unemployment, labour legislation.

\section{INTRODUCCIÓN}

El título de este trabajo puede parecer demasiado amplio y pretencioso y, desde luego si no se lee más que el título lo es. Sin embargo, en el desarrollo del artículo se va a comprender que los problemas son bastantes concretos, y que no tiene un horizonte infinito.

La primera parte del trabajo va a estar dedicada al análisis de algunos elementos de la Reforma Laboral del año 2012 y su incidencia causal en el desempleo, la precariedad, los bajos salarios, la inestabilidad y la rotación de los trabajadores en España.

La Reforma Laboral de 2012 llevada a cabo por el PP empieza con el Decreto Ley 3/2012 del 10 de febrero y que más tarde se modificó por la Ley 3/2012 del 6 de julio. En nuestro análisis vamos a fijarnos básicamente en este Decreto Ley y en la Ley.

Para introducir, aunque sea de una manera abusivamente esquemática este trabajo hay que decir que cuando entra en vigor el Decreto 3/2012 del 10 de febrero, y la Ley 3/2012 del 6 de julio, en el cuarto trimestre de este año, el número de parados alcanza la cifra de 5.965000 personas, la tasa de paro se sitúa en el 26,02 \% más o menos, y la de actividad en el 59.8 \% (www.ine.es). Cuatro años después (febrero de 2016) en este mes el número de parados en las oficinas públicas de empleo es de 4.152.986 desempleados y el desempleo aumentó en 2.231 desocupados en este mes. Sin embargo si se tiene en mente los datos de la EPA, que son los más exactos, el desempleo en España se eleva a casi 4.8 millones de desempleados, (El País 3 de marzo 2016). En febrero los contratos indefinidos firmados fueron 139.364 y superan el 10\%. A pesar de esto, la temporalidad es muy importante en 
cuanto que los que tienen un contrato indefinido suponen el $40.9 \%$ de los asalariados. Por último se puede aludir al informe de la Comisión Europea, (Informe sobre España 2016 SWD, del 26 de febrero 2016), que hace un diagnóstico en la página 50 sobre las políticas activas de empleo. Se dice que su capacidad es "limitada," que el servicio redujo su personal, que el número de ofertas sigue siendo bajo, que la cooperación entre agencias es marginal, que las agencias privadas dan servicio limitado, que no hay ningún seguimiento y que la garantía juvenil no está dando los frutos esperados.

Sin mitificar, ni mucho menos, los puntos de vista de la Comisión, que frecuentemente son más que discutibles, estas observaciones pueden dar que pensar. Dejamos aquí estas observaciones que van a servir de marco a discutir en las páginas que siguen.

El análisis de la producción legal sobre el trabajo entre 2012 y 2015 llenaría muchas páginas. Hay que referirse a uno de los muchos Decretos Ley que en este periodo han aparecido el RDL 16/2013 de 20 de diciembre "de medidas para favorecer la contratación estable y mejorar la empleabilidad de los trabajadores, agrega nuevas modificaciones legales del mercado laboral, que según el Decreto mismo ya constituían lo que se denomina aquí “La reforma Laboral” que se había iniciado con el RDL 3/2012.

A propósito de este Decreto, dicen Rodríguez Piñero, Valdés del Re y Casas Bahamonde (2014: 4), que "vuelve sobre reformas inmediatamente anteriores cambiando su signo a escaso tiempo de haber sido aprobadas, presentadas entonces como hallazgos para resolver los problemas de eficiencia del mercado de trabajo, en un tejer y destejer caracterizados de la "tensión reformista" instalada en nuestro ordenamiento laboral desde 2010". A continuación los autores se refieren a 8 decretos Ley y 6 leyes que han visto la luz en 2012 y 2013.

Es claro que es un número muy grande y que frecuentemente es difícil decir cuál es la finalidad de algunas y tampoco está del todo claro el plan reformador. Los autores que se han citado al principio continúan con una crítica que no es nada difícil de aceptar, "Las reformas, dicen, sobre unas mismas materias no siempre se complementan, sino que se desdicen, lo que dota al conjunto reformador, pese a lo pretendido, de una nada desdeñable fragilidad jurídica y de una sensación de provisionalidad que contradice el propósito proclamado de crear un marco jurídico estable y seguro que procure confianza a la empresa". La inflación de normas laborales para la constitución aluvional de un "Derecho de trabajo en deflación ha seguido distintos objetivos en nombre de la competitividad de la economía $y$ del empleo: flexibilidad del régimen de despidos y de la negociación colectiva, incentivos económicos a la contratación por tiempo determinado y con esto y en sustitución de esta, de la contratación a tiempo parcial y de la organización flexible del tiempo de trabajo. Todo ello sin consenso sobre el sentido de las reformas, ni sobre las técnicas y fórmulas jurídicas en que se materializan". 
Vamos a analizar únicamente algunos aspectos del Real Decreto Ley y únicamente nos vamos a referir a otras leyes sucesivas, cuando ello sea absolutamente necesario. La razón para esto es que el espacio es bastante limitado y el análisis de todas esas leyes y decretos llevaría consigo la necesidad de escribir un libro bastante voluminoso. Tampoco vamos a entrar en la relación que el Decreto Ley y la Ley tienen con respecto a los puntos de vista de la Comisión Europea. La Comisión tiene unos puntos de vista estrictamente neoliberales y estos instrumentos legales son en gran medida un destilado de neoliberalismo, pero la ideología del gobierno del Partido Popular es suficientemente neoliberal como para producir estas leyes y muchas más. De todas maneras, hay que decir que tanto el Sr. Luis de Guindos, Ministro de Economía, decía en la Comisión que se iba a publicar una Ley de Reforma "agresiva" y el Presidente del Gobierno, Sr. Rajoy, afirmó ante el Sr. Cameron, que cuando saliese le Ley se iba a llevar a cabo una huelga en España. En la disputa sobre la Reforma Laboral cuando el candidato del Partido Socialista se presentó más tarde a la investidura para Presidente del Gobierno, en su discurso sobre el programa dijo que derogaría la ley o leyes de la Reforma Laboral. También dijo que la ley había sido presentada en Europa como una especie de cumplimiento de las directrices europeas para convencer a la Comisión de que aceptase dar el préstamo necesario para el rescate de Bankia. Sea lo que sea parece que la Reforma laboral fue aprobada calurosamente por la Comisión Europea. Pero de todas maneras no vamos a detenernos en el análisis de esto. Como es obvio, no se puede analizar todo lo implicado en el Real Decreto y la ley de 2012 y por ello vamos a tratar de un modo telegráfico de 3 cuestiones:

1. El contrato de trabajo por tiempo indefinido de apoyo a los emprendedores;

2. La flexibilidad y sus dobles;

3. Los despidos colectivos.

\section{EL CONTRATO DE TRABAJO POR TIEMPO INDEFINIDO DE APOYO A LOS EMPRENDEDORES}

En primer lugar, queremos hacer unas consideraciones sobre lo que significan los emprendedores o el emprendedor. Aunque este término se considera algo neutral tiene una serie de connotaciones y denotaciones semánticas que hay que desentrañar.

Si tomamos la definición de emprendedor que ofrece el diccionario de la RAE se refiere a "una persona que emprende con resolución acciones dificultosas o azarosas," o una persona que acomete y comienza una obra, un negocio, un empeño. Este término se usa más comúnmente de los que encierran dificultades o peligros. Ampliando y concretando el campo semántico de emprendedor, se aplica a la persona que tiene iniciativa y decisión para acometer empresas, emprender negocios. El nombre de emprendedor denota creatividad individual para empezar u organizar una actividad comercial o industrial especialmente cuando incluye un riesgo financiero. David Harvey en un libro hoy ya 
clásico, afirma con razón que "el neoliberalismo es, en primer lugar, una teoría de prácticas de economía política que propone que el bienestar humano puede avanzar mejor liberando las libertades individuales de los emprendedores. Así como sus habilidades dentro de un marco institucional caracterizado por fuertes derechos de propiedad, mercados y comercio libres... La intervención del estado en los mercados, una vez que han sido creados, hay que mantenerla en el mínimo, dado que de acuerdo con la teoría no se puede poseer una información suficiente para analizar adecuadamente las señales de los mercados, y además grupos de intereses muy poderosos van a distorsionar y manipular las intervenciones del estado a su favor" (Harvey, 2005:2).

En el Capítulo II del RDL 3/2012, (artículos 4-7), se regulan una serie de medidas dirigidas al formato de la contratación indefinida y la creación de empleo... más adelante se podrá ver lo que esto quiere decir.

El artículo 4 del RDL recoge una novedad que es el contrato de trabajo por tiempo indefinido de apoyo a los emprendedores. Siguiendo con lo que se venía diciendo acerca del papel que la figura del emprendedor juega en la ideología y praxis neoliberal, hay que decir que esta figura de productor es un empresario, pero que tiene características propias que lo hacen una figura liminal entre el empresario y el trabajador autónomo y alejado de lo que es el trabajador asalariado.

La legislación se refiere con frecuencia al emprendedor que de alguna manera se convierte en una especie de protagonista. Es importante subrayar la aparición de la Ley 11/2013 de 26 de Julio de medidas de apoyo al emprendedor y de estímulo del crecimiento y de la creación de empleo.

Esta línea de política normativa sigue con una nueva ley: La 2 14/2013 de 27 de septiembre de apoyo a los emprendedores y su internacionalización.

La Ley 11/2013 dice que su objetivo es la creación y desarrollo de proyectos empresariales generadores de empleo y de valor añadido. En el preámbulo se afirma que "el apoyo a la iniciativa emprendedora, al desarrollo empresarial y a la creación de empleo es la lógica común que vertebra el conjunto de medidas que se recogen en esta ley".

Para ello, se propone conseguir "un alto grado de flexibilidad que permite ajustar los precios y salarios relativos". El concepto de flexibilidad que fue explicado de una manera muy amplia en el libro de Piore y Sabel (1986) y, por las mismas fechas, en la reforma laboral, promovida por el Gobierno de Felipe González en 1984, se explicó como una manera de superar la rigidez del sistema laboral español y hacerlo más flexible.

A partir de estas fechas la idea de la flexibilidad se instala como un elemento importante dentro de los países que han seguido el camino neoliberal y, ya se trate de la especialización flexible como de la acumulación, lo que han traído consigo son salarios bajos, inseguridad en el empleo y en muchos casos desempleo y pérdida de beneficios sociales y de la protección del trabajo. De ello hablaremos más adelante. 
La Ley 14/2013 acepta como un hecho fatídico "la falta de oportunidades de trabajo por cuenta ajena." En una situación como ésta hay que introducir la meta "del emprendimiento, del autoempleo y de la actividad empresarial para que los jóvenes alcancen las oportunidades de trabajo que el mercado de trabajo no les da y se autoempleen, y además, ellos mismos sean capaces de crear empleo....Todos estos elementos han de formar parte del sistema educativo," (Ley 14/2013), que traiga consigo "un cambio de mentalidad en el que se valore más la actividad emprendedora y la asunción de riesgos." Dentro del sistema educativo, hay un sistema de adoctrinamiento donde se imparta la "educación en el emprendimiento" para fomentar "la cultura del emprendimiento" y afianzar "el espíritu emprendedor y la cultura empresarial a partir de aptitudes como la creatividad, la iniciativa, el trabajo en equipo, la confianza en uno mismo y el sentido crítico". Se trataría de una especie de catecismo para fomentar algo que es en principio económico como el emprendimiento y la iniciativa empresarial que estén basados en aptitudes como la creatividad, la iniciativa del individuo y la autoconfianza. El neoliberalismo habría de ser trasmitido en la escuela. Estos principios del neoliberalismo se manifiestan de una manera palmaria en estas leyes que se refieren al emprendimiento, pero están presentes en todas las leyes y decretos que constituyen "la reforma laboral del partido popular".

Pero debemos volver al análisis concreto del contrato de trabajo por tiempo indefinido de apoyo a los emprendedores.

El contrato en cuestión, tiene como fin facilitar el empleo estable y potenciar la iniciativa empresarial. Cuando se analiza el régimen jurídico, lo primero que se instaura es un contrato con despido libre no indemnizado de un año de duración.

El nuevo contrato indefinido, que se celebrará a jornada completa, y se formalizará por escrito, solo puede concertarse por las empresas, cualquiera que sea su naturaleza jurídica, de menos de 50 trabajadores. En el preámbulo, se afirma que el 99,23\% de las empresas tienen estas características.

Es curioso que el régimen jurídico tiene una condición que es el establecimiento obligatorio "en todo caso" de un periodo de prueba con una duración de una año, también en todo caso. El establecimiento de un periodo de prueba tan largo está en contradicción con la doctrina judicial establecida. No se pueden "admitir como válidas fórmulas convencionales que pueden resultar incompatibles con la propia finalidad y funciones del instituto del periodo de prueba, pues una regulación convencional en la que se fije una duración excesivamente dilatada de dicho periodo, bien puede sugerir que se está dando amparo a un resultado que, en la práctica, puede asemejarse a la funcionalidad real de los contratos temporales, lo que podría encubrir situaciones de fraude de ley, al poder utilizarse para enmascarar unos fines no queridos por el citado precepto estatutario. Dichas cláusulas son radicalmente nulas por contrarias a la ley y al orden público y social" (Fernández, 2013: 130). 
Roberto Fernández, basándose en una sentencia del Tribunal Supremo de 20 de julio de 2011, da un golpe de gracia a la idea de que el empresario necesita un periodo de prueba tan largo como un año o dos. Cuando esto se introduce en leyes cuya finalidad es fomentar el empleo estable, la introducción de ese periodo de prueba vacía el fin de la ley y por ello el "contrato adquiere la funcionalidad de los contratos temporales. Con ello se está afirmando que de facto se trata de un contrato que favorece la temporalidad y también el desempleo.

La resolución o terminación del contrato durante este año de prueba es libre y sin indemnización (despido ad nutum como dicen los especialistas en Derecho del trabajo). En este periodo de prueba, no se aplica la protección de la contratación temporal, (no se aplica el régimen extintivo del contrato del trabajo y del despido, ni la indemnización de finalización de contrato, ni la presunción de indefinición por fraude de ley). Incluso en este periodo de un año los trabajadores tampoco tienen derecho al permiso retribuido de veinte horas anuales de formación. La contratación indefinida o estable se destruye con la inestabilidad e inseguridad que significa la prueba obligatoria de un año. Como muy bien subraya el Profesor Sala Franco (2012), "Se ha intentado justificar por la puerta de atrás la libertad total de despido durante los primeros doce meses de la relación laboral". Si lo que aquí aparece es el despido libre, nos encontraríamos con que lo que aquí se está defendiendo no es solo el neoliberalismo sino el más rancio liberalismo decimonónico. "Todo es más llamativo si además de esto se comprende que se trata de un contrato sometido a un larguísimo plazo de libre desistimiento para el empleador... que reduce a pura formalidad el carácter estable pretendido" (Molino Navarrete, 2012: 21), entonces se comprende perfectamente lo que muchos teóricos del Derecho del Trabajo han dicho con toda la razón, "este tipo de contratos ya son un clásico en toda reforma laboral que se precie y nunca han supuesto un aumento del índice de contratación ni tampoco una disminución de la temporalidad de las relaciones laborales, lo que ocurrirá también en esta ocasión. Pues la novedad más importante es que durante el primer año el despido es libre y gratuito" (Tomé Arnaiz, 2012: 17).

Quizás se puede pensar que hemos introducido un elemento que quizás no hayamos probado. Se ha afirmado que estos contratos "nunca han supuesto un aumento del índice de contratación" lo cual es lo mismo que decir que no han tenido una gran incidencia en la disminución del paro.

Para estudiar este problema hay que tener en cuenta los incentivos fiscales para este tipo de contratos.

Se aplica una deducción fiscal de 3000 euros en el supuesto de que sea el primer contrato de trabajo concertado por la empresa. 
- En el caso de contratar un desempleado beneficiario de prestación contributiva la empresa tiene derecho a una deducción fiscal de un 50 \% de la prestación por desempleo. También se dan bonificaciones:

- A jóvenes de 16 a 30 años durante 3 años:

1er año 1000 euros/año

$2^{\circ}$ año 1100 euros/año

3er año 1200 euros/año

- Mayores de 45 años:

1300 euros/año por 3 años /varones

1500 euros/año por 3 años /mujeres

No se aplica a este contrato la sanción a las empresas que extingan por despido reconocido o declarado improcedente de contratos bonificados. Esto supone el incentivo del despido con cargo a las cuotas de la Seguridad Social (Preciados Domenech, 2012).

Ante una situación como esta es fundamental tener en cuenta la observación de Casas Bahamonde. Rodríguez Piñero, Valdés del Re, (2012: 14). "En común tienen las medidas de fomento de la contratación el destino de fondos públicos, las bonificaciones de cuotas se financian con cargos a partidas presupuestarios del Servicio Público de Empleo Estatal, las reducciones de cuotas en los contratos para la formación y aprendizaje con cargo a la Tesorería General de la Seguridad Social. Esto sirve para aminorar el coste del trabajo para los empresarios sin que esas ((ayudas públicas)) hayan servido hasta el momento para asegurar efectos en la creación de empleo, ni siguiera en la reducción de desempleo de forma duradera, ni en el de la contratación temporal."

No podemos por menos de volver a citar los mismos autores en un editorial de Relaciones Laborales (2014: 4), "La política de empleo regresa de nuevo a las subvenciones públicas a la contratación, fuente indebida, pero ininterrumpida en nuestro ordenamiento, de creación o recreación de modalidades de contratación que, simultáneamente y contradictoriamente, se pretenden reducir. Por lo demás, los estudios realizados, ponen en duda la eficacia de estas medidas, también por la falta de instrumentos fiables para comprobar su efectividad, y por las distorsiones y desigualdades que generan en el mercado de trabajo, con el efecto adverso añadido de empobrecer los fondos de la Seguridad Social, lo cual a su vez se contrarresta, en esta norma reformadora y en otras, con la subida de las cotizaciones y la introducción de un factor de sostenibilidad en la determinación y cuantía de las pensiones de jubilación". Parece bastante claro, como dicen estos autores que las ayudas públicas a los empresarios no sirven para asegurar la creación de empleo, ni tampoco la reducción del desempleo ni la disminución de la contratación temporal. Pero, parece evidente que efectivamente 
son una transferencia de recursos desde los estratos más pobres de la población que, como es obvio, son los trabajadores que deberían ser ayudados en base a los recursos de la Tesorería de la Seguridad Social, a los grupos dominantes, los empresarios, sin que en principio esta tenga grandes resultados en la creación de empleo o en la mejora de su calidad. Con ello se consuma un fenómeno añadido de explotación estructural que viene favorecido por el Estado.

La finalidad de este contrato consiste en facilitar el empleo estable y favorecer la iniciativa empresarial. Cuando se examina el régimen jurídico del contrato lo que se instaura en primer lugar es un contrato con despido libre no indemnizado de un año de duración. Con ello aparece una contradicción. Se puede preguntar cómo es posible crear empleo estable si al principio del contrato que teóricamente llevaría a esa estabilidad, se instituye un periodo de un año en donde el despido no se indemniza y el contrato tiene como característica el despido libre. Parece una contradicción el despido libre y la estabilidad en el trabajo y sin embargo parece que hay gente que piensa que hay que vivir en la contradicción. Sin embargo hay otra contradicción que se ha mantenido durante mucho tiempo. Ya desde 1984 cuando se empieza a insistir y criticar la rigidez o falta de flexibilidad del mercado de trabajo se defendía una contradicción que se explicaba con una observación de lo que se podría designar "psicología pop." Los empresarios españoles contratan poco y no hacen contratos estables porque las condiciones para despedir los trabajadores son muy rígidas y costosas y, por ello, los empresarios no los contratan ni hacen contratos estables. Ni que decir tiene que esta idea es un sinsentido. Desde este punto de vista es conveniente traer a colación una observación de Molina Navarrete (2012: 14-15): "El Gobierno español - y con él muchos sectores de opinión y de presión - está convencido de que nuestro país necesita devaluarse para ser más competitivo.... y ha decidido hacerlo a través de una política que sí retiene, al menos formalmente...la de ordenación del mercado de trabajo nacional a través de la reducción de las condiciones de empleo y de trabajo. Naturalmente, ni puede socialmente ni quiere políticamente reconocer esta realidad, por lo que ha preferido construir un nuevo oxímoron, ya mantenido por el gobierno anterior y muy del gusto orwelliano....¿ Y por qué no iba el Gobierno a sostener que facilitando más el despido, a través de la objetivación de las causas y la reducción de sus costes se creará empleo?".

\section{LA FLEXIBILIDAD Y SUS DOBLES}

Un concepto que tiene una importancia fundamental en la constitución de la ley es el de la flexibilidad. La idea de la flexibilidad, o lo que se podía considerar como las demandas empresariales de flexibilidad, surgieron en España en la década de los 1980. Es especialmente importante a este respecto la ley 33/1984 con el PSOE en el poder. En ella se "descausaliza" el contrato de trabajo temporal como un método para "flexibilizar" el mercado de trabajo y con ello se instala en España lo que se ha llamado la "edad 
de oro del trabajo temporal" que dura hasta ahora mismo, (Martínez Veiga, 2015). En aquella época la idea de la flexibilidad circula mucho en los círculos políticos del PSOE y en los académicos (vid. Valdés del Re, 1985; Sala Franco, 1993). De todas maneras, este concepto de flexibilidad se convierte enseguida en una especie de emblema que, como todos los emblemas, es muy confuso. Es quizás Stephan Lessenich, (2015), el que mejor explica lo que este concepto significa. Según este autor, en los 1970 y 1980 las dos crisis del petróleo traen consigo una crisis de la idea del "bienestar para todos." En la época de Reagan y Thatcher tanto la economía como el estado buscan una reducción de costes y el aligeramiento de sus responsabilidades tratando de mejorar el mayor desarrollo de las mejores condiciones para los negocios y de la mayor libertad para los "sujetos económicos". Desde entonces la palabra mágica (utilizada ad nauseam) en las políticas económicas del mercado del trabajo ha sido la flexibilidad. Desde tasas de cambio flexibles a la selección flexible del lugar, desde la organización flexible del trabajo y la producción, horas flexibles de trabajo y de empleo hasta precios flexibles, salarios flexibles y derechos flexibles y, por fin, hasta la humanidad flexible. Ante la caída de los mercados globalizados, una cultura del éxito económico y el colapso del socialismo de estado, la flexibilidad se ha convertido en la quintaesencia del espíritu capitalista del postfordismo en el que cualquier rigidez se adscribe al "Imperio del mal".

En esta descripción de lo que representa la flexibilidad y su carácter totalizante, vago y confuso, y que, como veremos, puede tener como referente algo y su contrario, se comprenden perfectamente los problemas de la consideración de este término puede traer consigo. Pero, aunque parece evidente que la utilización del concepto de flexibilidad, realmente, nunca ha introducido claridad en el discurso, hay que tener en cuenta que las clarificaciones de tipo clasificatorio, a veces, parecen haber introducido claridad y otras veces oscuridad y confusión. De todas maneras, en un libro y clásico (Moody, 1997), se explica perfectamente lo que significa flexibilidad en la tradición del análisis del trabajo y la vez resume bien los diversos aspectos de la misma. Según él hay tres tipos de flexibilidad: funcional, numérica y temporal.

La flexibilidad funcional que se consigue con la eliminación de la descripción y demarcación entre trabajos, ya sea porque vienen del Taylorismo o de las negociaciones de los sindicatos. La flexibilidad funcional implica tareas múltiples y diversas, llevadas a cabo por los mismos actores.

La flexibilidad temporal implica operaciones a lo largo de todo el tiempo y parece usar los recursos del capital con la menor cantidad de trabajo posible. La flexibilidad del tiempo aparece en la rotación y en el uso masivo del tiempo extraordinario, a veces, sin pagarlo.

La flexibilidad numérica se refiere a la contratación externa, el uso del trabajo casual, trabajo temporal o a tiempo parcial. En última estancia significa el despido o la posibilidad del despido. 
Originariamente se consideraban dos tipos de flexibilidad: la funcional y la numérica. La flexibilidad funcional implicaba la posibilidad de cambio continuo en las tareas y la numérica el desempleo o el despido. Lo que estas dos realidades tienen en común se nos escapa. Realmente no tienen nada en común.

Esta clasificación de la flexibilidad que es bastante sencilla, aunque contradictoria se complica bastante en la ley 3/2012 de 6 de julio que en algunos momentos, parece un manual neoliberal de organización del trabajo. Quizás la complicación de las subdivisiones de la flexibilidad aparece en la obra de Wilthagen y Tror, (2004), en donde se trata de defender el concepto de "flexiseguridad" que aparece de pasada en el preámbulo de la ley y que ha tenido bastante éxito, aunque es un concepto confuso que, como se sabe, es un oxímoron, (Aparicio Tovar, 2012). De todas maneras, Wilthagen establece cuatro contenidos de la flexibilidad, (numérica externa, numérica interna, flexibilidad funcional y flexibilidad retributiva y otros cuatro conceptos de seguridad, (seguridad en el trabajo, seguridad en el empleo, seguridad en la renta - o en la seguridad social y combinación de seguridad).Aquí aparece una distinción fundamental entre flexibilidad interna y externa.

En la legislación española y también en las interpretaciones de los teóricos de Derecho del trabajo parece imponerse la distinción entre flexibilidad interna, y la flexibilidad externa que sería la que en otros contextos se llama numérica, (las extinciones e suspensiones del trabajo que llevan al desempleo).

En la historia de la legislación laboral Española hay dos leyes que están basadas en lo que se podría llamar “doctrina flexibilizadora." La primera ley es la de 35/2010 de 17 de septiembre que se puede designar como "Reforma Zapatero" y la segunda es la ley 3/2012 de 6 de julio que podemos designar como "Reforma Rajoy".

Es interesante la interpretación que las dos leyes ofrecen de la relación entre flexibilidad interna y externa. En el apartado II del preámbulo de la Ley 35/2010 se dice: "El capítulo II agrupa diversas medidas que pretenden potenciar los instrumentos de flexibilidad interna en el transcurso de las relaciones laborales, favoreciendo la adaptabilidad de las condiciones de trabajo a las circunstancias de la producción, bajo la consideración general de que dichos instrumentos constituyen una alternativa positiva frente a medidas de flexibilidad externa que implican un ajuste en el empleo.....entre estas medidas de flexibilidad interna se encuentran los relativos a los traslados colectivos, cláusulas de inaplicación salarial y, muy señaladamente la suspensión de contratos y reducción de jornada por causas económicas, técnicas, organizativas y de producción”.

En el preámbulo de la Ley 3/2012 se dice en su apartado IV que "el capítulo III agrupa diversas medidas para favorecer la flexibilidad interna en las empresas como alternativa a la destrucción de empleo. El problema de la dualidad laboral es consecuencia, en buena medida, de un sistema de instituciones laborales inadecuado que ha quedado evidenciado 
durante la última crisis. En un sistema que genera incentivos adecuados, las empresas pueden hacer frente a las oscilaciones de la demanda recurriendo a mecanismos diferentes al despido, que preservan el capital humano de la empresa, tales como reducciones temporales de salario o de jornada. Este tipo de ajuste ha sido relevante en los países de nuestro entorno, lo que se ha traducido en una menor destrucción de empleo".

En los dos textos, (el de la ley de 2010 y de la 2012), aparece una continuidad muy llamativa. De alguna manera se pretende justificar el aumento de la flexibilidad interna, que se introduce en estos textos normativos, con el argumento de que ello evitará el recurso de las empresas a una medida más traumática como es el despido. Sin embargo, hay un cambio de matiz en la ley de 2012 cuando apuesta por el equilibrio entre la flexibilidad interna y la externa, entre la regulación de la contratación indefinida y la temporal, la de la movilidad interna en la empresa y la de los mecanismos extintivos del contrato de trabajo. Mientras que en la ley de 2010 se defiende que las medidas de flexibilidad interna son una alternativa positiva frente a medidas de flexibilidad externa (ajuste en el volumen de empleo). Al menos en el texto de la ley de 2010 se afirma que son preferidas las medidas de flexibilidad, mientras que en la ley 2012 se apuesta por el equilibrio entre flexibilidad interna y externa.

De todas maneras, Alarcón Caracuel (2014: 49) explica muy bien el problema de las relaciones o pretendidas relaciones entre la flexibilidad interna y externa. Tanto en la ley de 2010 como en la de 2012, "se pretende justificar el aumento de la flexibilidad interna que estos textos normativos introducen con el argumento de que ello evitará el recurso a una medida más traumática como es el despido. La falacia del argumento se comprueba inmediatamente: basta leer ambas leyes para comprobar que las dos contienen preceptos dedicados a facilitar los despidos: no hay, pues un intercambio entre flexibilidad interna y flexibilidad externa puesto que aumentan los dos. Y el resultado práctico ya lo conocemos los despido no han hecho más que aumentar desde 2010, y han aumentado a partir de 2012 de tal forma que hemos llegado a más de los 6 millones de parados en el país".

\section{LOS DESPIDOS}

Efectivamente entre 2010 y el momento actual, el primer trimestre de 2016, (el momento en que escribimos), el paro registrado no ha bajado del $21 \%$. Con ello hay que decir que "España sufre su periodo más largo con el paro, por encima del 20\%," (El País 29 de abril de 2016). El paro de larga duración se reduce un poco pero sigue en 2,7 millones de personas, la mayoría sin prestación y en estado de pobreza severa. Desde la Reforma Laboral del 2012 se ha destruido un millón de empleos de jóvenes. Las previsiones del Gobierno parecen un poco demasiado optimistas. Según él en 2016 la tasa de paro se va de 19,9\%, en 2017 de 17,9\% en 2018 de 15,8\% y en 2019 de $14 \%$.

La coincidencia entre este largo periodo de tasa de desempleo del $21 \%$ que va de 2010 a 
2016 y el pico de aumento que comienza en 2012 con más del 25\% y sube a casi el 27\%, precisamente cuando se implanta la ley de 2012 debe hacernos pensar que no se trata de un fenómeno casual sino que la legislación tiene claramente un influjo en este fenómeno. (En el gráfico que ponemos a continuación esta tomado de El País 29 de abril de 2016: $35)$.

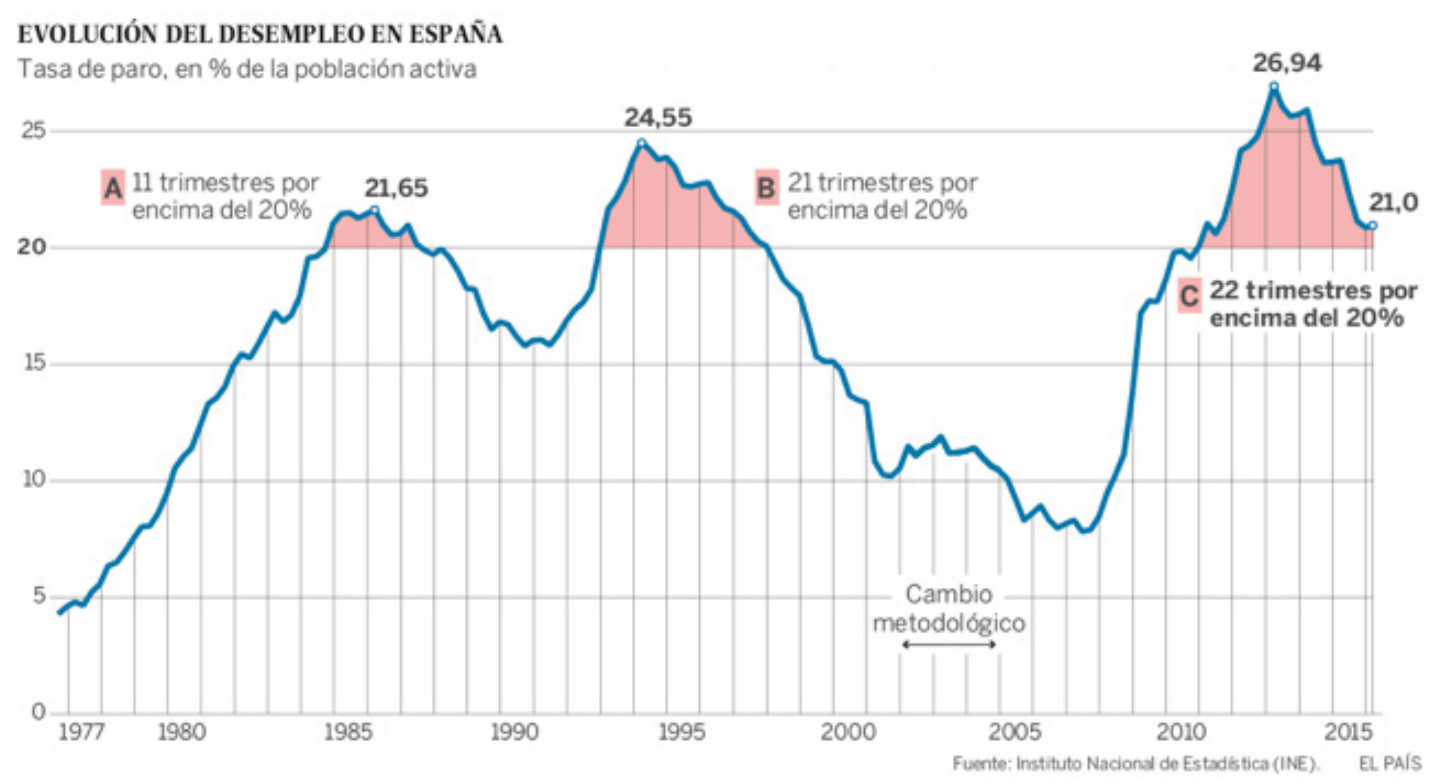

Fig.1

Lo que parece claro de todas maneras es que a pesar de que en algunas de estas reformas se insiste en la idea de la flexibilidad interna como sustitutiva de la flexibilidad externa, el mercado de trabajo español sigue caracterizándose por la continua rotación, tanto en su vertiente de gestión ordinaria, (altísima tasa de temporalidad), como en su vertiente de gestión extraordinaria que se sigue realizando en base al despido o a técnicas que se podían denominar como técnicas extintivas. Hace años que yo mismo llevé a cabo un trabajo en El Ejido, en abril de 2000, en el que se preguntaba a más de una treintena de trabajadores inmigrantes básicamente marroquíes, sobre cuánto tiempo habrían trabajado para el mismo patrón en lo que iba de año, y la media de acuerdo con las respuestas era de 15 días.

Lo que aquí se produce es una rotación y movilidad entre los diversos patrones. Esta movilidad y rotación es una característica básica del mercado de trabajo español que persiste (Martínez Veiga, 2004).

Otro ejemplo mucho más reciente puede darnos una indicación de lo que este fenómeno significa. El País del lunes 2 de mayo de 2016: p.3 trae una noticia de interés. Se titula "Los sindicatos denuncian la elevada temporalidad del personal del Canal". Según los Sindicatos en el Canal de Isabel II no hay un procedimiento normalizado de selección de personal. Como consecuencia de ello el personal contratado no es el adecuado. Desde julio de 2012 a septiembre de 2015, se habrían hecho unas 600 contrataciones y todas ellas temporales. 
Los directivos del Canal están de acuerdo en que los contratos temporales han aumentado mucho en los últimos años, y dicen que "se da una prohibición de llevar a cabo contratos indefinidos" y se apoyan para esto "en la normativa vigente." La gran cantidad de temporales contratados ha traído consigo una enorme precariedad laboral con unos sueldos muy bajos, "según los datos ofrecidos por los sindicalistas, desde 2012 a 2016 se ha producido una rotación de alrededor de 1500 personas". Dentro del Canal está la planta potabilizadora de Valmayor, que es una las mejores de Europa, y en la que el 99 \% de los trabajadores son temporales.

Los sindicalistas dicen que "a los trabajadores se les forma y cuando ya están preparados se les despide porque están en prácticas y así está estipulado en la ley”. Por último los sindicatos piensan que los problemas, que existen en la contratación, se resolverían si se recuperase el Convenio Colectivo que perdieron cuando la Comunidad creó la nueva sociedad de Isabel II Gestión en 2012, (en pleno vigencia de la ley del mismo año). "Utilizando el cambio jurídico como argumento" pierde vigencia el XVIII convenio de empresa y se aplica el de ámbito estatal.

Las medidas de flexibilidad interna que en principio parecería que deberían ser estructurales en la organización del trabajo se convierten en características que tienen una agencia, que no es más que el propio empresario. Casas Bahamonde, Rodríguez Piñero y Valdés dal Re (2012: 17) hacen una observación sobre la ley del 2012 que puede parecer exagerada pero que es bastante exacta. Según ellos, en esta ley se "intenta reinstalar en nuestro sistema jurídico la concepción de la empresa como un territorio de exclusiva gestión por el empresario rescatando del baúl de la memoria, donde le había colocado la cláusula constitucional del Estado social y democrático de Derecho, la figura del empresario como el Señor de su casa, (Herr im Hause), figura esta ligada a concepciones autoritarias de los sistemas de relaciones laborales".

Las palabras en alemán son bastantes llamativas para demostrar cómo, "el nuevo régimen jurídico refuerza, sin disimulo alguno, los poderes empresariales, cuyo ejercicio no ha sido moderado ni atemperado por una paralela vigorización de los derechos de participación de los trabajadores. Antes al contrario, tales derechos han sido mermados, de modo que, por ilustrar la aseveración con un sencillo ejemplo, un empresario con un censo de trabajadores de los que dos son ejecutivos, podrá reducir la cuantía salarial de 9 de ellos sin otro requisito formal que la simple notificación de la decisión modificativa al delegado de personal," Casas Bahamonde, Rodríguez Piñero y Valdés dal Re (2012: 20).

Lo que ha llevado a cabo la Reforma Laboral de la ley 3/2012 es incrementar las potestades empresariales de gestión de la mano de obra. Como muy bien señalaba Falguera Baró (2012: 277), "con la Reforma de 2012 se ha producido un desplazamiento sensible de la línea de equilibrio del modelo de relaciones laborales español hacia el ámbito de los intereses empresariales". Por ello, aunque en este trabajo se ha tratado de poner 
en relación esta ley con desarrollos legales anteriores, no cabe duda que representa una ruptura con ellos. "Es muy significativo el incremento de los poderes empresariales en el contrato de trabajo, mayores competencias en materia de despidos colectivos, mayores capacidades de modificación sustancial de las condiciones de trabajo, ruptura de la eficacia vinculante del convenio.... Se pretende, de otra parte, promover un concepto unidireccional de flexibilidad, exclusivamente basado en necesidades productivas y eliminando tutelas, obviando la necesidad de dotar a la misma de un carácter bilateral, (nueva regulación de las suspensiones de contratos y reducciones de jornada, de la movilidad funcional, de las modificaciones sustanciales de trabajo, ruptura, de la movilidad geográfica), y se potencia el incremento de la precarización contractual, (nuevo contrato indefinido de apoyo a los emprendedores, el enésimo cambio de contrato de aprendizaje o la adquisición de la condición de agencias de colocación de las ETT" (Falguera Baró, 2012).

La protección jurídica frente al despido injustificado ha venido sufriendo en cada una de las reformas de la legislación laboral de los últimos años una lenta y progresiva relajación que ha afectado a las causas para despedir, a las exigencias de forma, al control público o sindical, así como a los remedios que el juez puede arbitrar. No puede ignorarse que esa política legislativa ha sido permeable a concepciones de la empresa y del ejercicio de la autoridad en la misma medida que tienden a una desvalorización del trabajo subordinado a favor de una ideología económica que exalta el beneficio empresarial. Aparicio Tovar (2012), dice "la aceptación de la contratación temporal indiscriminada que hizo la reformas de 1994 y su mantenimiento "de facto" posterior tiene como efecto práctico una grave erosión de la protección frente al despido...... la relajación que la legislación, pero sobre todo la jurisprudencia, ha hecho de la exigencia de la causa para despedir". Con ello se quiere decir que se busca la causa para indemnizar más que la causa para despedir. Como dice Rodríguez Piñero (2009), se opera en la práctica como un despido libre ad nutum indemnizado, que puede tener el mismo coste para el empresario, sea un despido justificado o injustificado, con indiferencia de que se haya ejercido arbitraria o regularmente, de buena o mala fe el poder empresarial.

De todas maneras, es importante volver a plantear el problema de la flexibilidad, porque en ella está el núcleo del problema. Hay que subrayar que a partir de la Reforma Laboral de 1994 se intenta ofrecer un planteamiento equilibrado de las diversas formas de flexibilidad. En la Exposición de motivos de la ley 11/1994 se propone "equilibrar el reparto de fórmulas de adaptabilidad o flexibilidad entre las diversas fases del desarrollo de la legislación laboral con el propósito de favorecer una adecuación flexible de la gestión de los recursos humanos en la empresa tanto a la situación económica de la misma como a los cambios en el mercado de trabajo."

A pesar de la búsqueda de ese equilibrio entre la flexibilidad interna y externa, esta fue incapaz de alterar la preferencia empresarial por las fórmulas de flexibilidad externa. 
Después de la Reforma de 1994 se crea en 1997 el "contrato para el fomento de la contratación indefinida cuyo atractivo básico era la reducción de la indemnización por despido objetivo improcedente, o de la puesta en circulación en 2002 de lo que posteriormente se iba a llamar despido "exprés" con la eliminación de la obligación de los salarios de tramitación en caso de despido improcedente.

El tema de la flexibilidad interna aparece claramente en la ley 25/2010 y en la ley 3/2012.

En la ley 35/2010, dentro de la Exposición de motivos, se presenta la flexibilidad interna "como mecanismo de utilización preferente frente al recurso a la destrucción de puestos de trabajo".

En la ley 3/2012, en su Exposición de motivos se subraya la importancia de introducir medidas para "favorecer la flexibilidad interna en las empresas como alternativa a la destrucción de empleo". Con una cierta sorna pero muy exactamente afirma Sanguinetti Raymond (2012) que el favorecimiento de la flexibilidad interna como alternativa a la externa, en la ley $3 / 2012$ es "un objetivo imposible de conseguir, como es evidente, mientras esta última (flexibilidad externa / destrucción de empleo) se vea facilitada y abaratada por el propio legislador".

En una comunicación muy importante, Guerrero Vizete (2012) ha insistido en que la reforma de 2012 tiene visos de dirigirse en dirección contraria a la pretendida, dado que no frena la contratación temporal y flexibiliza el despido, por lo que terminará siendo el gran obstáculo para la estabilidad en el empleo.

Cuando se analiza el funcionamiento del mercado de trabajo y los resultado de la ley $3 / 2012$, se descubre que lo que realmente ocurre es que cuando se intenta fomentar la utilización preferente de los mecanismos adaptativos de la flexibilidad interna, realmente es necesario encarecer y dificultar los mecanismo extintivos o de flexibilidad externa, así como la penalización por la utilización masiva e injustificada de los contratos temporales. Como muy bien subraya Alfonso Mellado (2010), "si no hay mecanismos de motivación y penalización las crisis productivas y de actividad seguirán acometiéndose por medio de ajuste de plantillas".

En segundo lugar hay que tener en cuenta que la flexibilidad interna queda reflejada como un instrumento de actuación frente a la crisis de empresa porque la extinción se lleva a cabo utilizando la figura del despido exprés. Este tipo de despido se ha seguido utilizando (por medio del reconocimiento de la improcedencia y la puesta a disposición de la cuantía necesaria), y esto tiene lugar aún después de que el despido exprés ha sido suprimido por la ley $3 / 2012$.

Como dicen Casas Bahamonde, Rodríguez Piñero y Valdés dal Re, (2012: 1), la reforma de la ley $3 / 2012$ está basada en las reformas precedentes. "Pero llega más lejos superando las 'líneas rojas' que el anterior gobierno habría trazado especialmente en lo relativo a los 
costes del despido y al mantenimiento de la autorización administrativa para los despidos $y$ suspensiones colectivas de los contrataos de trabajo. Ahora se produce un debilitamiento de los mecanismos de negociación colectiva de la adaptabilidad de relación tanto con las medidas de flexibilidad interna como con las deflexibilidad externa, aliviando notablemente los costes del despido".

Lo que se acaba de decir nos lleva a plantear el problema del despido y especialmente del despido improcedente. Parece claro que en la legislación laboral española más reciente se ha procedido al abaratamiento del mismo a golpe de reformas.

Se trata de las siguientes (vid. Ballester Pastor, 2013: 306-309): En primer lugar se produce la supresión de los salarios de tramitación por medio del despido exprés cuando se reconoce la improcedencia y se paga la indemnización, (Ley 45/2002). También se disminuye el coste de las indemnizaciones por despido improcedente en la introducción del fomento de empleo, (Ley 12/2001 y Ley 43/2006).

Por último, se produce la eliminación de los salarios de tramitación en caso de despido improcedente cuando no se opta por la readmisión, y se disminuye de modo generalizado el despido improcedente que de 45 días de salario por año de servicio con el límite de 45 mensualidades pasa a ser de 33 días de salario por año de servicio con el límite de 24 mensualidades. Esto último se introduce en la Ley 3/2012. En esta misma ley se propone el contrato indefinido de apoyo a los emprendedores con un periodo de prueba de un año en el que el despido es libre.

Cuando se asume como normal la indemnización del despido improcedente como coste inevitable aparejado a la extinción por crisis, no se desincentiva el acceso a la contratación temporal, cuya única sanción cuando se lleva a cabo sin causa es precisamente el coste del despido improcedente. El coste del despido no es la causa de la contratación temporal porque sea alto, como se afirma frecuentemente, sino porque es demasiado bajo.

Al final de nuestras observaciones es importante volver sobre el problema del despido.

La ley de 2012 interviene por una parte sobre lo que se puede considerar coste del despido. Esto se lleva a cabo al reducir las indemnizaciones por despido improcedente, incluso el disciplinario, a 33 días de salario por año de trabajo con un máximo de 24 mensualidades. Se ha hecho impracticable la readmisión de los trabajadores en despidos improcedentes, cuando esta posibilidad está abierta, al imponer, únicamente en este caso, el pago de los salarios de tramitación, y eliminándolos cuando el trabajador opta por la indemnización. También ha actuado en segundo lugar sobre el despido por causas relativas a la empresa. Con ello parece ser que se intenta ajustar el volumen de empleo a los cambios técnicos organizativas operados en ella. Con ello se intentaban corregir las disfuncionalidades de la legislación anterior. Desde los años 80 se decía que las disfuncionalidades eran la existencia de una gran "flexibilidad de entrada" en el trabajo y al mismo tiempo "una 
rigidez de salida" que consistía en que el procedimiento duraba mucho y además se exigía la autorización administrativa para despedir, y esto da lugar a negociaciones que a veces terminan aumentando las indemnizaciones. Esto se critica en la Exposición de motivos de la le 3/2012. La Reforma de 2012 se presenta como basada en un equilibrio entre flexibilidad interna y flexibilidad externa. Desde este punto de vista hay que decir que se trata de un equilibrio entre contratación indefinida, y contratación temporal, entre movilidad funcional dentro de la empresa y los mecanismo extintivos en el contrato de trabajo, entre las tutelas operantes en el contrato de trabajo y en el mercado de trabajo.

En la ley se suprime el contrato para el fomento de la contratación indefinida. También se generalizan en todos los contratos la indemnización reducida por despido, con esta generalización pierde su sentido el contrato de fomento cuyo elemento fundamental era la indemnización más pequeña.

La ley de 2012 no cambia nada sobre la contratación temporal y mantiene su amplitud que era protegida por muchos años.

De todas maneras parece conveniente observar cual es el comportamiento de los empresarios. "La Encuesta Anual Laboral” en su segunda edición (2011), da unas pistas interesantes y el economista José Manuel Lago Peñas y Jesús Cruces Aguilera hacen un análisis muy interesante de ella, (2016: 27-29). De él tomamos algunas observaciones centrales:

El primer dato es llamativo: Solo el 10\% de los empresarios han empleado medidas de reordenación, (flexibilidad) interna (salarial o de jornada), frente al 25\% que aplicó el ajuste duro, los despidos y la no renovación de contratos temporales. Según la encuesta, las empresas que aplican la flexibilidad interna y externa son el 14\% del total. Pero el análisis en detalle de este agregado refleja que lo que hizo la gran mayoría de estas empresas fue ajustar el empleo con medidas duras mientras que las de flexibilidad interna fueron simplemente un acompañamiento. Por último, en la Encuesta se pregunta qué harían las empresas ante una caída de la actividad. La respuesta del 62\% de las empresas es: reducir costes laborales despidiendo trabajadores temporales.

Un elemento fundamental es la intención, dentro de la ley, de aligerar el proceso de toma de decisiones en empresas que consiste en la desaparición de la exigencia de la autorización administrativa en las reducciones de jornada y suspensión del contrato por causas empresariales. También se atribuye al empresario una nueva facultad unilateral según la cual se podrá establecer la distribución irregular de la jornada de trabajo. El texto legal dice que "en defecto de pacto, la empresa podrá distribuir de manera irregular a lo largo del año el diez por ciento de la jornada de trabajo." Parece claro que se atribuye al empresario una nueva, (entre otras muchas), facultad importante como es distribuir el $10 \%$ de la jornada del trabajo que como muy bien calcula Alarcón Caracuel (2014: 50), 
"nótese que el 10 \% de la jornada laboral de 1800 horas son 180 horas, más de un mes de jornada laboral que el empresario podrá distribuir a su antojo".

Además, si se tiene en cuenta que la movilidad funcional que se refiere básicamente al poder empresarial de ordenar al trabajador que deje de hacer una cosa y haga otra, se puede comprender que el poder del empresario aumenta mucho.

También hay que hacer alusión a la movilidad geográfica. En primer lugar, "tanto el traslado como el desplazamiento del trabajador se deciden por voluntad unilateral del empresario. Es cierto que en ambos supuestos, el empresario deberá fundamentar su decisión en razones económicas, técnicas, organizativas o de producción. Pero no es menos cierto que la concurrencia será valorada exclusivamente por el empresario y su orden deberá ser acatada por los trabajadores afectados sin necesidad de acuerdo por su parte" (Alarcón Caracuel, 2014: 52).

Por último hay que aludir a lo que Alarcón Caracuel (2014: 59) dice acerca de "la manifestación más potente del poder empresarial es la de alterar las condiciones laborales inicialmente pactadas con sus trabajadores: la modificación sustancial de las condiciones de trabajo.

Las condiciones laborales que pueden modificarse sustancialmente se pueden resumir en tres elementos: la jornada, (horario y distribución del tiempo del trabajo), salario, (sistema de remuneración y cuantía salarial), y funciones. Además también hay que incluir el sistema de trabajo y rendimiento. Aparecen aquí los elementos de flexibilidad interna en la ordenación y, en el cambio de ellos la determinante es el poder del empresario.

En el análisis de la flexibilidad interna hay que partir de una observación básica de Molero Marañón (2012: 369) "la facilitación empresarial sin paliativos de las medidas de flexibilidad interna se duda seriamente que de forma inmediata o en tiempo de crisis vaya a frenar los mecanismos de flexibilidad de salida, a la luz de las reformas adoptadas en este ámbito,.....el incremento de la utilización de las medidas de flexibilidad interna no se producirá mirándose solo a si mismo sino que se promoverá en la medida en que se reduzca el abuso en la utilización de la contratación temporal y se recurra de forma menor a las vías de ajuste que proporciona el despido. Las medidas de flexibilidad interna y externa no funcionan como compartimentos estancos en el mercado de trabajo, sino que las últimas se han utilizado de manera desmesurada en detrimento de las promesas. Hasta la fecha, las medidas de flexibilidad de salida han sido los recursos adicionales de ajuste en tiempo de crisis por las estructuras empresariales y la última reforma en lugar de dificultar su utilización o frenar su recurso abusivo, las favorece sin pudor alguno, facilitando intensamente el despido económico y la contratación a término".

Da la impresión de que uno de los objetivos fundamentales de esta "reforma" como de otras leyes anteriores consiste en dar un mayor poder al empresario en las relaciones de 
trabajo. La potencia que adquiere este poder se incrementa por la intervención legislativa en dos puntos esenciales. Se trata de la intervención legislativa, tanto en la negociación colectiva al permitir un descuelgue masivo, al prever un arbitraje obligatorio ante las discrepancias en la aplicación del convenio, al limitar su ultractividad y al dar prioridad al convenio de empresa frente al sectorial. El segundo punto, que es el que más nos va a interesar en este momento, son las modificaciones en el régimen del despido, de las que ya hemos hablado pero que se van a resumir ahora. En primer lugar se ha limitado el coste del despido para el empresario al reducir la indemnización por despido improcedente e incluso en el disciplinario reduciéndola a 33 días de salario y a un máximo de 24 meses. También se ha actuado sobre el despido por causas relativas al funcionamiento de la empresa para "ajustar el volumen de empleo a los cambios técnicos-organizativos operados en las empresas.

La aceptación de la contratación temporal indiscriminada produce una gran erosión en la protección contra el despido. Es también grave la relajación que ha llevado a cabo la jurisprudencia de la causa para despedir, de tal manera que si se ha producido una desnaturalización de su función porque los jueces tienden a "despreciar el elemento causal con base en la compensación económica del trabajador despedido injustificadamente" (Ramos Quintana, 2009: 18). Más que para despedir buscan la causa para indemnizar lo que para algunos significa aceptar el despido libre pero indemnizado. Esto "opera en la práctica como un despido libre "ad nutum" indemnizado, que puede tener el mismo costa para el empresario sea un despido justificado o injustificado, con indiferencia de que se haya ejercido arbitraria o regularmente, de buen o mala fe el poder empresarial," (Rodríguez Piñero, 2009: 11). Se podría pensar que el olvido del elemento causal en los despidos y su intercambio por la indemnización siempre reducida es algo que siempre los facilita y favorece.

Esta relajación de las condiciones del despido es lo que produce claramente el aumento del mismo y por ello, consecuentemente, el desempleo. Desde este punto de vista habría que distinguir claramente la creación de empleo y el despido. También se puede pensar que "el desarrollo de las leyes del trabajo nunca ha servido de facto para crear empleo", como han afirmado muchos autores, "las normas sociales, contra aquello que reiteradamente se afirma, no crean empleo per se" algo reconocido por el propio gobierno, eso sí, después de aprobar el Real Decreto Ley, (en donde se decía lo contrario) (Falguera Baró, 2012). Se puede defender con toda razón que las leyes del trabajo nunca han servido para la creación de empleo pero en este caso han servido para favorecer el despido y claramente para crear desempleo. 


\section{BIBLIOGRAFÍA}

Alarcón Caracuel, Manuel R. (2014) La alteración de las condiciones de trabajo inicialmente pactadas: Diversas Instrumentos Legales tras la Reforma Laboral de 2012. En Nuria Pomar Beltrán (coord.) La flexibilidad interna en los despidos colectivos en el sistema español de relaciones laborales. Madrid / Barcelona: Huygens, pp. 43-63.

Aparicio Tovar, Joaquín (2012) "Las causas del despido basadas en necesidades de Funcionamiento de la Empresa”. Revista de Derecho Social 57, pp.145-164.

Ballester Pastor, María Amparo (2013) "La flexibilidad interna en el marco de las leyes laborales". En Asociación Española de Derechos del Trabajo y de la Seguridad Social (comp.) Las reformas del derecho del trabajo en el contexto de la crisis económica: la reforma laboral de 2012. Valencia: Tirant lo Blanc, pp. 279-396.

Casas Bahamonde, María Emilia; Rodríguez-Piñero y Bravo-Ferrer, Miguel y Valdés dal Ré, Fernando (2012) “La Nueva Reforma Laboral”. Relaciones laborales: Revista crítica de teoría y práctica 5: 1-39.

Falguera Baró, Miguel Ángel (2012) “El Real Decreto Ley 3/2012: más ideología que empleo". Jurisdicción Social 117: 210-280.

Fernández, Roberto (2013) "El nuevo contrato indefinido de apoyo a emprendedores: luces y sombras". En Asociación Española de Derechos del Trabajo y de la Seguridad Social (comp.) Las reformas del derecho del trabajo en el contexto de la crisis económica: la reforma laboral de 2012. Valencia: Tirant lo Blanc, pp. 127-147.

Harvey, David (2005) A Brief History of Liberalism. Oxford: Oxford University Press

Lago Peñas, José Manuel y Cruces Aguilera, Jesús (2016) “Estrategias de los interlocutores sociales”. En Confederación Sindical de CCOO (ed.) Cuadernos de Acción Sindical. El impacto de la reforma laboral de 2012 en la negociación colectiva. Mayo, pp. 27-40.

Lessenich, Stephan (2015) "Mobility and Control: On the Dialectic of the Active Society". En Klaus Dörre, Stephan Lessenich, y Hartmut Rosa (ed.) Sociology, Capitalism, Critique, London: Verso, pp. 98-142.

Martínez Veiga, Ubaldo (2005) Trabajadores invisibles: precariedad, rotación y pobreza de la inmigración en España. Madrid: La Catarata.

Martínez Veiga, Ubaldo (2015) "Spain's labour reforms: temporary work, flexibility and disciplining the working class". En Victoria Goddard y Susana Narotzky (eds) Industry and Work in Contemporary Capitalism. Global Models, Local Lives. Oxford: Routledge, pp. 169-180.

Mellado, Alfonso (2010) "Las actuaciones para incentivar la flexibilidad interna". En VVAA (ed.) La Reforma Laboral en la ley 15/2010. Valencia: Tirant Lo Blanc, pp. 95-120. 
Molero Marañón, María Luisa (2012) ·La flexibilidad interna promovida por las sucesivas reformas laborales". En Rodríguez-Piñero y Bravo-Ferrer, Miguel y Valdés dal Ré, Fernando (coord.) La Reforma Laboral de 2012 en materia de negociación colectiva. Las Rozas: La Ley, 309-380.

Molina Navarrete, Carlos (2012) "De las reformas laborales a un nuevo e irreconocible estatuto del trabajo 2012. Comentario Sistemático al Real Decreto Ley 3/2012 de reforma del mercado de trabajo". Estudios financieros. Revista de trabajo y seguridad social 348, p. 7-184.

Moody, Kim (1997) Workers in a Lean World. London: Verso.

Piore, Michael y Sabel, Charles (1986) The Second Industrial Divide, Nueva York: Basic Books.

Preciados Domenech, Carlos Hugo (2012) Una Primera Aproximación al Real Decreto Ley 3/2012 de Reforma Laboral. Albacete: Bomarzo.

Ramos Quintana, Manuel (2009) "Debates en torno al despido en España y amortización objetiva y colectiva de puestos de trabajo". Revista de Derecho Social 47, pp. 13-38.

Rodríguez-Piñero y Bravo-Ferrer, Miguel; Valdés dal Ré, Fernando y Casas Bahamonde, María Emilia (2014) "Contratación a tiempo parcial y flexibilidad del tiempo de trabajo en la nueva reforma laboral (RDL 16/2013, de 20 de diciembre)". Relaciones laborales: Revista crítica de teoría y práctica 2, pp. 1-27.

Rodríguez-Piñero y Bravo-Ferrer, Miguel (2009) "Sobre el contrato de trabajo único". Relaciones laborales: Revista crítica de teoría y práctica 1, pp. 11-24.

Sala Franco, Tomas (1993) "El debate sobre las políticas de flexibilidad laboral y el Derecho del Trabajo". En VVAA (comp.) La flexibilidad laboral en España. Universidad de Zaragoza, Zaragoza, pp. 62-75.

Sala Franco, Tomas (2012) "Los juristas avisan de que la reforma va cambiar seniors por juniors”. Cinco Días, 1 de marzo.

Sanguinetti Raymond, Wilfredo (2012) "La reforma flexibilizadora de las modificaciones sustanciales de las condiciones de trabajo y sus límites inmanentes”. Revista de Derecho Social 57, pp. 125-144.

Tomé Arnaiz, Antonio (2012) "La reforma del mercado laboral: nada equilibrada y extremadamente agresiva". Iuris 169, pp. 16-19.

Valdes dal Re, Fernando (1985) "Flexibilidad en el mercado de trabajo y ordenamiento laboral”. Papeles de Economía Española 22, pp. 302-315.

Vizete, Guerrero (2012) "Estabilidad en el empleo y reforma laboral: la debilitación de un principio". XXIII Jornadas Catalanes de Dret Social. 8 y 9 de marzo. Universitat Oberta de Catalunya (UOC). 
Wilthagen, Thom y Tror, Francis (2004) "The concept of flexibility: a new approach to regulating employment and labour markets". Transfer, European Review of Labour Research 10(2), pp. 166-186. 